\title{
Direct optoelectronic generation and detection of sub-ps-electrical pulses on sub-mm-coaxial transmission lines
}

\author{
Tae-In Jeon ${ }^{\text {a) }}$ and D. Grischkowsky ${ }^{\text {b) }}$ \\ School of Electrical and Computer Engineering, Oklahoma State University, Stillwater, Oklahoma 74078
}

(Received 23 July 2004; accepted 25 October 2004)

\begin{abstract}
We report efficient direct optoelectronic generation of sub-ps- $\mathrm{THz}$ pulses on $50 \Omega$ coaxial transmission lines with a $330 \mu \mathrm{m}$ diameter solid copper outer tube filled with Teflon containing the $80 \mu \mathrm{m}$ diameter inner conductor. The transmitted pulses after propagating as much as $105 \mathrm{~mm}$ were measured at the end of the line with an optoelectronic antenna having sub-ps-time resolution. We observed low-loss, single transverse electromagnetic mode propagation with very little group velocity dispersion. The experimentally derived values for the frequency-dependent absorption are consistent with the theoretical predictions. (C) 2004 American Institute of Physics.
\end{abstract}

[DOI: 10.1063/1.1839645]

Recently, efficient broadband coupling of freely propagating pulses of $\mathrm{THz}$ electromagnetic radiation into circular and rectangular metal waveguides has been demonstrated. ${ }^{1,2}$ Single-mode coupling and propagation were achieved for these waveguides, and thereby demonstrated much larger bandwidths with $1 / 10$ the loss of lithographically defined coplanar transmission lines. ${ }^{3}$ Although these waveguides are quite useful for narrow band or $\mathrm{THz}$ time-domain spectroscopy (THz-TDs) applications, they all have very high group velocity dispersion (GVD), which make them incapable of sub-ps-pulse propagation.

The excessive broadening of sub ps $\mathrm{THz}$ pulses by these waveguides is caused by the extreme GVD near the cutoff frequencies. ${ }^{1-3}$ This excessive pulse broadening would not occur for the transverse electromagnetic (TEM) modes of a two-wire transmission line, a coaxial transmission line, or a metal parallel-plate waveguide (PPWG), that do not have a cutoff frequency. The group and phase velocities of these TEM modes are determined solely by the surrounding dielectric. Unfortunately, quasioptic coupling techniques are not effective for the complex field patterns of the TEM modes of the two wire or the coaxial transmission lines.

However, efficient coupling is possible for the simple field pattern of the TEM mode of the metal PPWG and has been recently reported. ${ }^{4}$ In a more recent demonstration, $0.22 \mathrm{ps} \mathrm{THz}$ input pulses to a flexible $\mathrm{Cu}$ PPWG were observed to broaden only to $0.39 \mathrm{ps}$ after propagating $250 \mathrm{~mm}{ }^{5}$ The minimal broadening of the output pulses was due to the absorption of the higher frequency components of the pulse by the waveguide due to the finite conductivity of $\mathrm{Cu}$. Consequently, a THz interconnect capable of propagating sub-ps-pulses with minimal loss and little distortion has been realized. ${ }^{3}$

Here, in the continuing search for the ideal $\mathrm{THz}$ interconnect, we report the first optoelectronic generation of subps-THz pulses launched directly into commercial sub-mmdiameter coaxial transmission lines and the optoelectronic measurement of the propagated pulses. These $\mathrm{THz}$ pulses were then used to characterize the frequency response of the

\footnotetext{
${ }^{a}$ Permanent address: Division of Electrical and Electronics Engineering, Korea Maritime University, Busan, Korea.

b)Electronic mail: grischd@ceat.okstate.edu
}

coaxial lines by THz-TDS from 0.05 to $1 \mathrm{THz}$, the broadest bandwidth and highest frequency characterization of coaxial transmission lines to date.

For completeness we note the earlier work on the transfer of shorter than 5 ps pulses to and between coplanar and coaxial devices, ${ }^{6}$ and the optoelectronic characterization of a microwave probe up to $400 \mathrm{GHz}^{7}$ Also, the related and unusual observations of guided ps pulse propagation on the single wire of a near-field optical probe have been reported. ${ }^{8}$

The experimental setup is different from that used previously, ${ }^{1-5}$ because here the sub-ps-THz pulses were directly generated on the coaxial transmission line, using a new method for which the photoconductive material is brought into physical contact with the biased microstructure. As shown in Fig. 1, the inner conductor of the coaxial line is biased at $-120 \mathrm{~V}$ with respect to the outer conductor. The photoconductive silicon on sapphire (SOS) chip has the $0.5-\mu \mathrm{m}$-thick silicon face in contact with the biased inner and outer conductors of the coaxial line on the polished flat input face. Ultrafast $40 \mathrm{fs}, 800 \mathrm{~nm}$ incoming laser pulses at a repetition rate of $83 \mathrm{MHz}$ and an average power of $88 \mathrm{~mW}$ illuminate the SOS to generate pulses of photoconductors. These carriers are accelerated by the strong radial elecric field from the line and thereby generate $\mathrm{THz}$ pulses, well matched to the propagating TEM mode. The coupled THz pulses propagate through the line and are measured at the output by an ion-implanted SOS THz antenna, for which the silicon side faces the polished output face of the coaxial line, covered by a $55-\mu \mathrm{m}$-thick polyethylene tape for insulation from the bias voltage. The transient electric fields are detected by driving the antenna with the sampling laser pulses, as a function of the relative time delay between the excitation and sampling pulses. ${ }^{1-5}$

The measured propagated pulses through the $15 \mathrm{~mm}$ long, $44 \mathrm{~mm}$ long, and $105 \mathrm{~mm}$ long coaxial transmission lines are shown in Figs. 2(a)-2(c). For the shortest $15 \mathrm{~mm}$ propagation the measured full width at half maximum (FWHM) output pulsewidth is $0.87 \mathrm{ps}$, as indicated on Fig. 2(a); for $44 \mathrm{~mm}$ the pulsewidth is $1.15 \mathrm{ps}$, and for $105 \mathrm{~mm}$ the pulsewidth is $1.56 \mathrm{ps}$. These relatively short pulsewidths are comparable to those generated on coplanar transmission lines. ${ }^{3}$ However, the pulse attenuation with propagation is much less than for coplanar lines and is comparable to that 
(a)
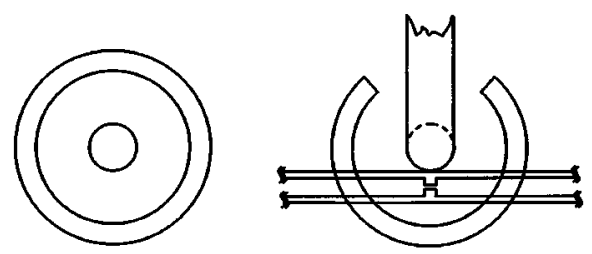

(b)

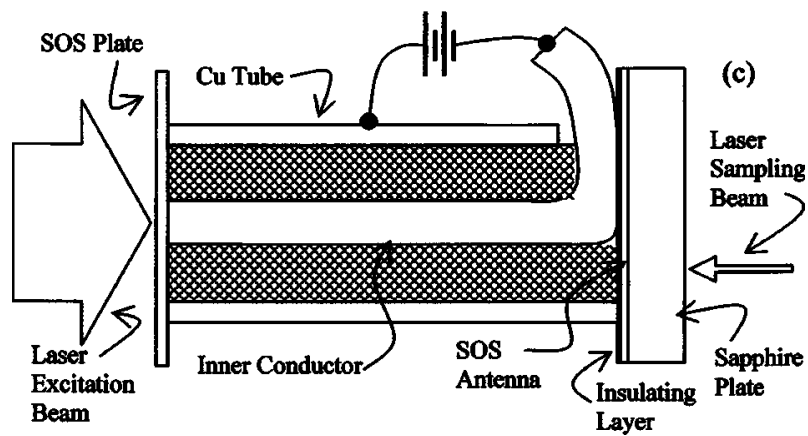

FIG. 1. (a) The input face of the coaxial transmission line showing the outer $\mathrm{Cu}$ tube with $330 \mu \mathrm{m}$ outside diameter and $260 \mu \mathrm{m}$ inside diameter, the PTFE (Teflon) dielectric, and the $80 \mu \mathrm{m}$ diameter Au plated W inner conductor. The cable was from Coax Co., Ltd. Yokohama, Japan with Part No. SC-033/50, design impedance $50 \Omega$, and maximum single mode frequency $0.392 \mathrm{THz}$. (b) the output face shows the central conductor extended outside of the line for biasing. The optoelectronic antenna is also shown; (c) the experimental schematic shows the SOS excitation plate in contact with the input face of the coaxial line, shown in cross section. The biasing is shown together with the positioning of the insulating film, the SOS antenna and the additional $0.5 \mathrm{~mm}$ sapphire plate, used to extend the time between the transmitted and reflected THz pulses. The dielectric is shown cross-hatched.

achieved for the single conductor waveguides ${ }^{1,2}$ and metal PPWG. ${ }^{4,5}$ The desired TEM propagation is shown by the small broadening observed upon propagation, mainly due to the attenuation of the high frequency components, ${ }^{4,5}$ although there is a small GVD due to the dielectric. The structure observed on the first 20 ps of the trailing edges is considered to be reflective ringing on the output due to both the bent and extended central conductor used for biasing and the sapphire plate.

The corresponding pulse spectra are shown in Figs. 3(a) -3 (c) for the coax lengths of 15,44 , and $105 \mathrm{~mm}$, respectively. The large feature spectral oscillations are due to the complicated features on the trailing edge of the pulse. The rapid oscillations observed in Figs. 3(a) and 3(b) indicates the onset of the $\mathrm{TE}_{11}$ mode. ${ }^{1,2}$ The longer path for Fig. 3 (c) has effectively attenuated the $\mathrm{TE}_{11}$ mode with higher propagation loss. When the Fourier transform of the output pulses is only performed on the initial section of the transmitted pulse out to the times indicated by the arrows on Fig. 2 , the lower resolution frequency spectra indicated by the heavy dashed lines in Figs. 3(a)-3(c) are obtained and are used in the calculations of absorption.

The propagation losses for the TEM mode due to the finite conductivity $\sigma$ of the metal can be expressed in terms of an amplitude absorption constant $\alpha_{c}$ given by ${ }^{9,10}$

$$
\alpha_{c}=0.5(1+b / a) R n_{d} /\left[\eta_{o} b \ln (b / a)\right],
$$

where $n_{d}=1.43$ is the index of refraction of Teflon (PTFE), $b=130 \mu \mathrm{m}$ is the inside radius of the Cu tube, $a=40 \mu \mathrm{m}$ is the radius of the inner conductor, $\eta_{o}=377 \Omega$ is the impedance of free space. The characteristic resistance $R=10.88$ $\times 10^{-3} \sqrt{10^{7} / \sigma \lambda_{o}}$, will be in $\Omega$, if $\sigma$ is in $1 / \Omega \mathrm{m}$ and the free-space wavelength $\lambda_{o}$ is in meters. For the TEM mode

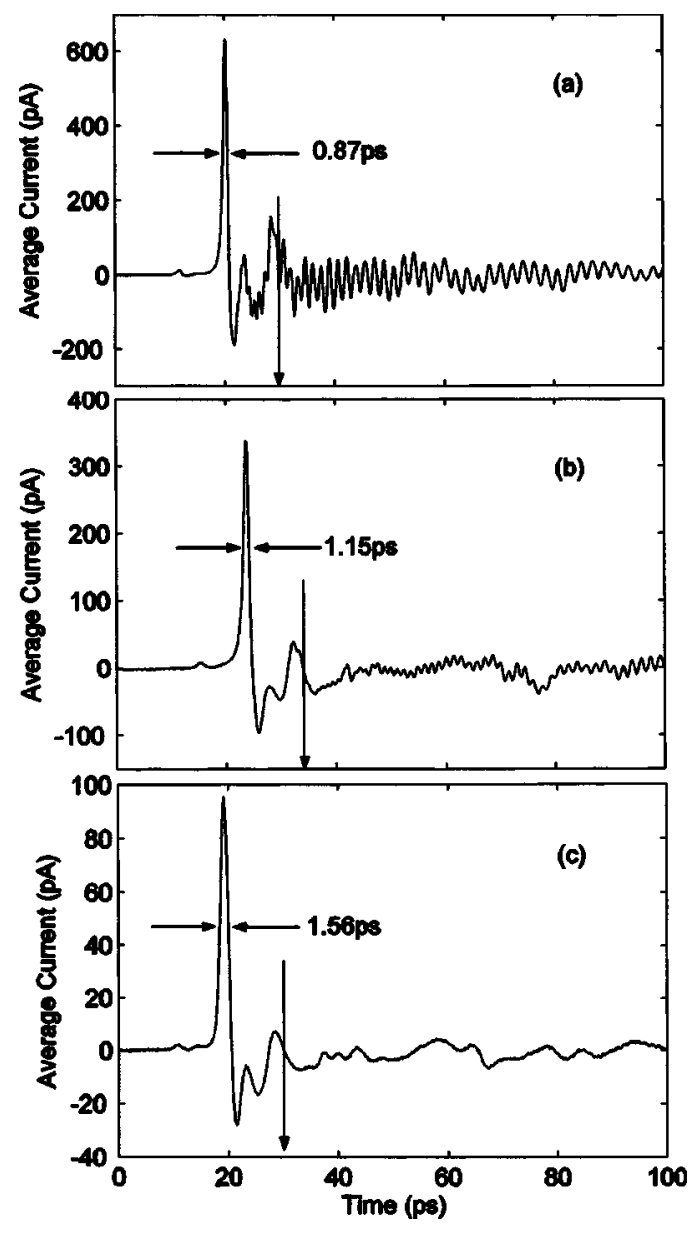

FIG. 2. Measured output $\mathrm{THz}$ pulses (a)-(c) for the coaxial transmission line lengths of 15,44 , and $105 \mathrm{~mm}$, respectively. The arrows indicate the truncated pulses used to obtain the simplified Fourier transforms.

the additional amplitude absorption constant $\alpha_{d}$ due to the dielectric is the same as for a freely propagating electromagnetic wave. The total TEM amplitude absorption coefficient is given by $\alpha_{t}=\alpha_{c}+\alpha_{d}$.

It is informative to compare with the absorption coefficient of the metal PPWG given by $\alpha_{c}^{P P}=n_{d} R /\left(\eta_{o} h\right)$, where $h$ is the plate separation. ${ }^{5}$ Again, the absorption loss $\alpha_{d}$ in the dielectric is the same as for a freely propagating wave. Although the coaxial line solves the problem of the beam divergence in the unguided dimension of the PPWG, it requires a dielectric to support the inner conductor. Even if the dielectric did not absorb $\mathrm{THz}$ radiation, $\alpha_{c}$ would be increased, because it is proportional to $n_{d}$. However, it is relatively easy to have an air-spaced parallel plate waveguide and to thereby eliminate this loss. Consequently, the observed loss for the coaxial transmission line is significantly higher than for a comparable air-spaced PPWG due to both $\alpha_{d}$ and $n_{d}$.

Figure 4 presents the calculated $\alpha_{c}$ using Eq. (1) for the $330 \mu \mathrm{m}$ diameter coaxial line, assuming a $\mathrm{Cu}$ inner conductor. Figure 4 also presents $\alpha_{d}$ from our THz-TDS characterization of Teflon, which is consistent with earlier work, ${ }^{11}$ but is more precise. The total absorption coefficient $\alpha_{t}=\alpha_{c}+\alpha_{d}$ is also presented. For an additional comparison $\alpha_{\xi}^{P P}$ is shown for the air spaced, Cu PPWG with $h=90 \mu \mathrm{m} .{ }^{5}$ It is to be noted that the radial separation of the conductors of the $330 \mu \mathrm{m}$ diameter coaxial transmission line is also $90 \mu \mathrm{m}$, and that $\alpha_{c} / n_{d}=1.25 \alpha_{c}^{P P}$, showing that if the coaxial transmission line could be air spaced the total loss would be only to AIP license or copyright, see http://apl.aip.org/apl/copyright.jsp 


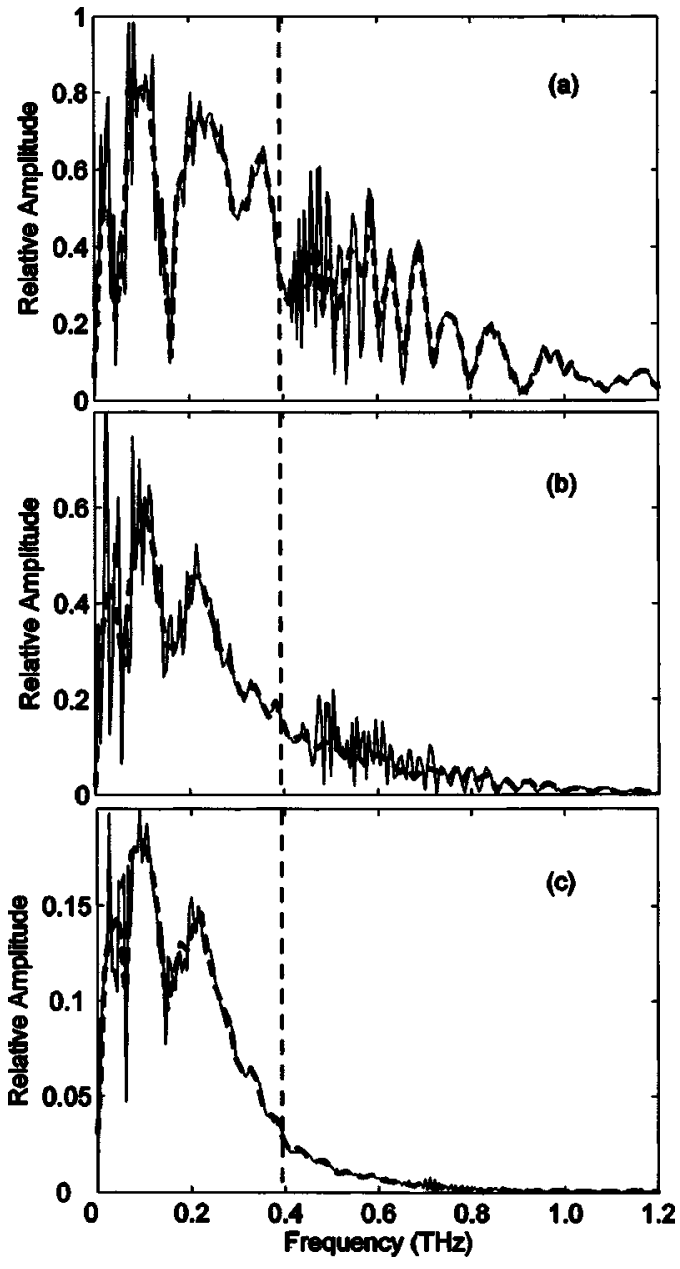

FIG. 3. (a)-(c) Corresponding amplitude spectra of the THz pulses shown in Figs. 2(a)-2(c) respectively. The heavy dashed line spectra are for the truncated pulses of Figs. 2(a)-2(c). The light vertical dashed lines indicate the cut-off frequency of the $\mathrm{TE}_{11}$ mode.

$25 \%$ more than for the PPWG. For our coaxial line approximately one half of the observed loss is due to the metal and the other half is due to the dielectric.

The experimental amplitude absorption is obtained from the simple relationship $A(\omega)=A_{o}(\omega) \exp \left[-\alpha_{t}(\omega) L\right]$, for which $A_{o}(\omega)$ is the TEM mode amplitude spectrum coupled into the coaxial line, $L$ is the line length and $A(\omega)$ is the measured output amplitude spectrum. Thus, it is straightforward to determine $\alpha_{t}(\omega)$ from the ratios of the three (dashed lines) amplitude spectra of Fig. 3. In Fig. 4, we plot $\alpha_{t}(\mathrm{c} / b)$ and $\alpha_{t}(\mathrm{c} / a)$ determined from the ratios of the measured spectral amplitudes $A_{\mathrm{c}}(\omega) / A_{b}(\omega)$ and $A_{\mathrm{c}}(\omega) / A_{a}(\omega)$, from Figs. 3(a)-3(c), respectively. The scatter of these two determinations gives an indication of our measurement accuracy. The experimental problems mainly responsible for this scatter are the differing input pulses to the three different length lines and the differing receiver response. We do not understand why the measured absorption is significantly higher than theory, although this was also the case for the PPWG. ${ }^{5}$

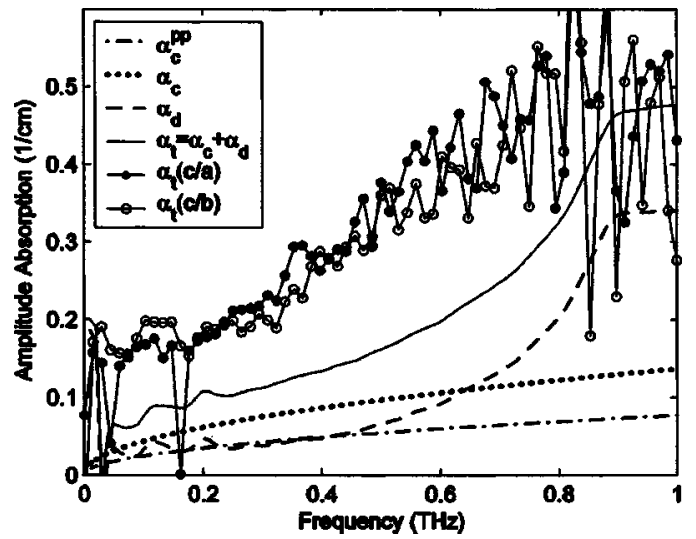

FIG. 4. Measured total amplitude absorption coefficient $\alpha_{t}$ (c/a, dots) and $\alpha_{t}$ (c/b, open circles) compared to the theoretical absorption $\alpha_{t}$. The theoretical absorption coefficient of the coaxial line $\alpha_{c}$ and the measured Teflon $\alpha_{d}$ are also shown. For comparison the theoretical absorption $\alpha_{c}^{P P}$ is shown for the air-spaced, Cu PPWG. (Ref. 5).

Lower conductivity metal and higher absorption in the dielectric are possibilities. Nevertheless, the agreement with theory is acceptable at this stage and shows that the system is understood.

In summary, we have characterized a sub-mm-coaxial transmission line from 0.05 to $1 \mathrm{THz}$, using direct optoelectronic generation of sub-ps-THz pulses on the line and highbandwidth optoelectronic detection of the output pulses. For $15 \mathrm{~mm}$ propagation we observe $0.87 \mathrm{ps}$ (FWHM) output pulses, which broaden to $1.56 \mathrm{ps}$ with $105 \mathrm{~mm}$ propagation. Our measurements are consistent with theory and show that for frequencies below the $\mathrm{TE}_{11}$ cutoff, a sub-mm-coaxial line is a practicable TEM-THz interconnect with minimal pulse distortion and loss.

This work was partially supported by the National Science Foundation, the U.S. Army Research Office, and the Semiconductor Research Corporation, Center for Advanced Interconnect Systems Technologies.

${ }^{1}$ R. W. McGowan, G. Gallot, and D. Grischkowsky, Opt. Lett. 24, 1431 (1999).

${ }^{2}$ G. Gallot, S. P. Jamison, R. W. McGowan, and D. Grischkowsky, J. Opt. Soc. Am. B 17, 851 (2000).

${ }^{3}$ D. Grischkowsky, IEEE J. Sel. Top. Quantum Electron. 6, 1122 (2000).

${ }^{4} \mathrm{R}$. Mendis and D. Grischkowsky, Opt. Lett. 26, 846 (2001).

${ }^{5}$ R. Mendis and D. Grischkowsky, IEEE Microw. Wirel. Compon. Lett. 11, 444 (2001)

${ }^{6}$ M. Bieler, M. Spitzer, H. Lecher, G. Hein, and U. Siegner, Electron. Lett. 38, 125 (2002).

${ }^{7}$ M. Bieler, M. Spitzer, H. Lecher, G. Hein, and U. Siegner, IEEE MTT-S Dig. 3, 1811 (2003).

${ }^{8}$ K. Wang, A. Barkan, and D. M. Mittleman, Appl. Phys. Lett. 84, 305 (2004).

${ }^{9}$ N. Marcuvitz, Waveguide Handbook (Peter Peregrinus, London, 1993), Chap. 2.

${ }^{10} \mathrm{P}$. A. Rizzi, Microwave Engineering-Passive Circuits (Prentice Hall, Upper Saddle River, NJ, 1988).

${ }^{11}$ J. R. Birch, E. A. Nicol, D. V. Bartlett, and T. P. Hughes, Infrared Phys. Technol. 36, 799 (1995). 